\title{
Synthesis, Evaluation of Pharmacological Activity, and Molecular Docking of 1,4-Dihydropyridines as Calcium Antagonists
}

\author{
Moataz Ahmed Shaldam, ${ }^{* a}$ Mervat Hamed El-Hamamsy, ${ }^{b}$ Dalia Osama Saleh, ${ }^{c}$ and \\ Tarek Fathy El-Moselhy ${ }^{b}$ \\ ${ }^{a}$ Department of Medicinal Chemistry, Faculty of Pharmacy, Delta University for Science and Technology; Gamassa \\ 35712, Egypt: ${ }^{b}$ Department of Pharmaceutical Chemistry, Faculty of Pharmacy, Tanta University; Tanta 31527, \\ Egypt: and ${ }^{C}$ Department of Pharmacology, National Research Center; Dokki 12622, Egypt. \\ Received September 28, 2015; accepted January 5, 2016; advance publication released online January 27, 2016
}

1,4-Dihydropyridine (DHP) is an important class of calcium antagonist. It inhibits the influx of extracellular $\mathrm{Ca}^{2+}$ through L-type voltage-dependent calcium channels. Two series of nifedipine analogues were synthesized and evaluated as calcium antagonists. The ortho-nitrophenyl ring of nifedipine was replaced with an ortho- or a meta-chlorophenyl substituent. The $\mathrm{IC}_{50}$ values revealed that some of the compounds are similar to or more active than nifedipine. Substitution with groups of suitable bulkiness, such as ethyl ester, at the 3- and 5-positions of the DHP ring gave $3 \mathrm{~h}$, which is approximately three-fold more active than nifedipine as a calcium antagonist. A docking study with the DHP receptor model was performed to interpret the differences in calcium antagonist activities. The molecular docking study demonstrated that the lipophilicity of the substituted phenyl group at the 4-position of the DHP ring is an important factor that could increase the activity of the calcium antagonist taking the steric factor into consideration. Bulky groups interfere with ring-to-ring hydrophobic interaction with $\mathrm{Tyr}^{1460}$ and limit the efficiency of increasing the length of the hydrocarbon chain of esters at the 3- and 5-positions of the DHP ring as an approach to increase activity. The presence of a chelating substituent on the phenyl ring at the 4-position of the DHP ring may ensure strong binding to the receptor and hence stabilization of the closed-channel conformation.

Key words 1,4-dihydropyridine (DHP); calcium channel blocker; pharmacological evaluation; synthesis; molecular docking

Calcium is essential in all living organisms. Different types of calcium channels can control the influx of calcium ions into cells. ${ }^{1)}$ The influx of calcium ion into the cell takes place in both normal functioning and in pathologic processes. ${ }^{2,3)}$ This raises the importance of calcium channels as targets for many drugs. Various drugs can modulate either agonist or antagonist, the calcium ion entry through L-type $\mathrm{Ca}^{2+}$ channels. ${ }^{4-7)}$ Calcium antagonists have a various pharmacological activity including antihypertensive and antianginal agent, ${ }^{8-10)}$ antitumor, ${ }^{11)}$ anti-inflammatory, ${ }^{12,13)}$ antitubercular, ${ }^{4)}$ analgesic ${ }^{14)}$ and antithrombotic. ${ }^{15,16)}$

1,4-Dihydropyridines (DHPs) bind selectively to L-type calcium channel protein. $\left.{ }^{17}\right)$ They can promote either channel activation or inhibition. ${ }^{5,18)}$ DHP calcium antagonists yet developed are vasodilators. ${ }^{19)}$ Vasodilatation is due to the uncoupling of the contractile mechanism of vascular smooth muscle, which requires $\mathrm{Ca}^{2+} \cdot{ }^{20}$ )

Several studies that targeted structure-activity relationship (SAR) of DHP action at the L-type channel have focused on the importance of the lipophilicity of DHPs in increasing calcium antagonist activity. ${ }^{21-24)}$ Chlorophenyl ring is a lipophilic group that can be introduced in 4-position of the DHP ring and hence increases the lipophilicity of the target compounds. In this research, ortho- and meta-chlorophenyl rings in 4-position of the DHP ring was used. Also side chain esters in 3- and 5-positions were designed to increase lipophilicity to investigate lipophilic effect on calcium antagonist activity.

\section{Results and Discussion}

Chemistry Two series of DHP derivatives were synthe- sized. A series of symmetric achiral DHPs (3a-k) has been synthesized by using classical Hantzsch condensation ${ }^{25)}$ as shown in Chart 1. The asymmetric chiral DHPs series (6a-n) has been synthesized by a modified Hantzsch reaction as described by Meyer et al., ${ }^{26)}$ as shown in Chart 1 . The synthesized compounds have either 2-chlorophenyl or 3-chlorophenyl ring at the 4-position of the DHP ring. All structural features necessary to reserve pharmacological activity were considered. The preparation, collection and purification of the products were carried out in the absence of oxidizing agents and in darkness. The elemental analysis results for the synthesized compounds were within $\pm 0.4 \%$ of the calculated values and the ${ }^{1} \mathrm{H}-\mathrm{NMR}$ spectra for the synthesized compounds in $\mathrm{CDCl}_{3}$ were compatible with the assigned structures. The structures of test compounds were shown in Fig. 1.

Pharmacology All synthesized compounds were subjected to evaluating their intrinsic calcium antagonist activity using high $\mathrm{K}^{+}$contraction guinea-pig ileal longitudinal smooth muscle (GPILSM) method. ${ }^{27-30)}$ This method depends on the elevation of cytosolic $\mathrm{Ca}^{2+}$ concentration upon high potassium-induced membrane depolarization through DHP-sensitive calcium channels. ${ }^{31,32)}$

The $\mathrm{IC}_{50}$ values were extracted from the graph using Graphpad Prism V6.01 software. A triplicate experiment was performed for each compound. The calculated standard error of the mean (S.E.M.) reflected non-significant variation in the results. The results were presented as $\mathrm{IC}_{50} \pm$ S.E.M. in Table 1. These results were tested statistically for significance using one-way ANOVA following post hoc test in SPSS 21 software. The results were statistically significant $(p=0.000)$. This indi- 


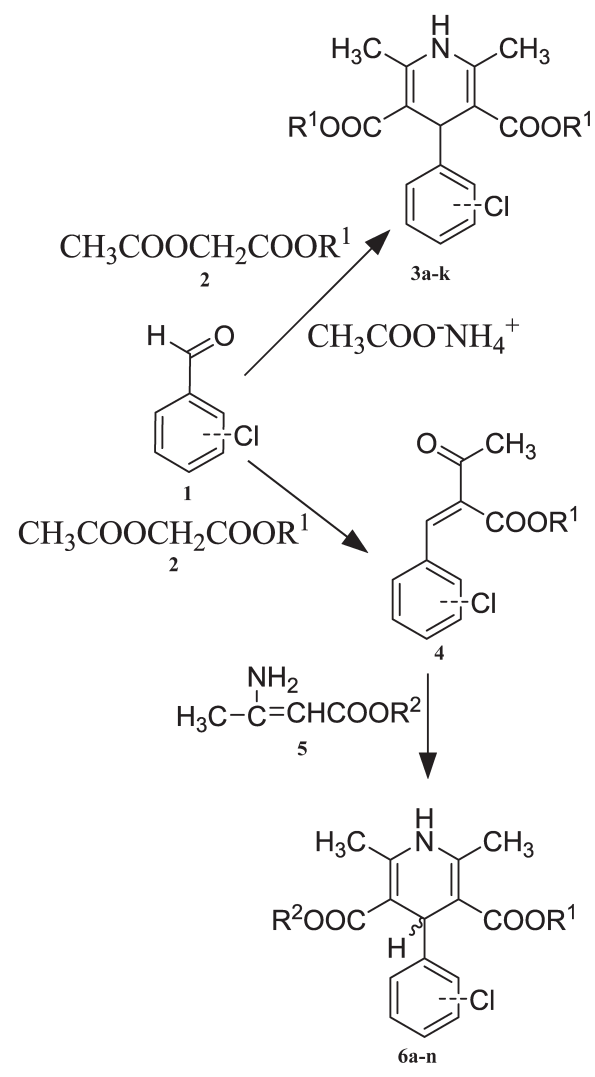

Chart 1. Synthesis of Symmetrical Compounds (3a-k) and Asymmetrical Compounds $(\mathbf{6} \mathbf{a}-\mathbf{n})$<smiles>[R20]OC(=O)C1=C(C)NC(C)=C(C(=O)O)C1(C(=O)O[R20])c1ccccc1[X]</smiles>

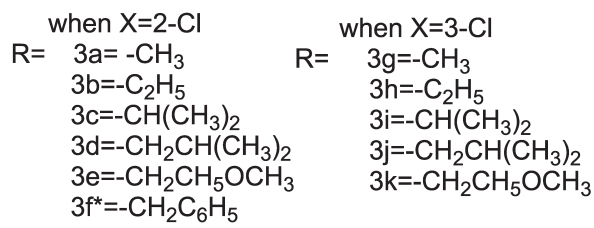

Table 1. Pharmacological Activity of the Synthesized Compounds and Nifedipine as a Reference Compound

\begin{tabular}{|c|c|}
\hline Compound No. & $\mathrm{IC}_{50}( \pm \text { S.E.M. })^{a)}$ \\
\hline $3 a$ & $4.27 \times 10^{-10}( \pm 0.114)$ \\
\hline $3 \mathbf{b}$ & $2.00 \times 10^{-10}( \pm 0.037)$ \\
\hline $3 \mathrm{c}$ & $4.02 \times 10^{-10}( \pm 0.002)$ \\
\hline 3d & $5.44 \times 10^{-10}( \pm 0.192)$ \\
\hline $3 e$ & $2.07 \times 10^{-10}( \pm 0.006)$ \\
\hline $3 f$ & $8.57 \times 10^{-10}( \pm 0.058)$ \\
\hline $3 g$ & $4.88 \times 10^{-10}( \pm 0.020)$ \\
\hline $3 \mathrm{~h}$ & $1.59 \times 10^{-10}( \pm 0.112)$ \\
\hline $3 \mathbf{i}$ & $4.10 \times 10^{-10}( \pm 0.123)$ \\
\hline $3 \mathbf{j}$ & $7.00 \times 10^{-10}( \pm 0.061)$ \\
\hline $3 k$ & $4.32 \times 10^{-10}( \pm 0.131)$ \\
\hline $6 a$ & $2.39 \times 10^{-10}( \pm 0.119)$ \\
\hline $6 b$ & $3.55 \times 10^{-10}( \pm 0.253)$ \\
\hline $6 c$ & $4.83 \times 10^{-10}( \pm 0.087)$ \\
\hline $6 d$ & $7.76 \times 10^{-10}( \pm 0.151)$ \\
\hline $6 e$ & $5.87 \times 10^{-10}( \pm 0.073)$ \\
\hline $6 f$ & $2.68 \times 10^{-10}( \pm 0.133)$ \\
\hline $6 \mathrm{~g}$ & $7.21 \times 10^{-10}( \pm 0.106)$ \\
\hline $6 \mathrm{~h}$ & $6.98 \times 10^{-10}( \pm 0.012)$ \\
\hline $6 \mathbf{i}$ & $6.01 \times 10^{-10}( \pm 0.041)$ \\
\hline $6 \mathbf{j}$ & $2.81 \times 10^{-10}( \pm 0.116)$ \\
\hline $6 \mathrm{k}$ & $2.71 \times 10^{-10}( \pm 0.084)$ \\
\hline 61 & $5.97 \times 10^{-10}( \pm 0.056)$ \\
\hline $6 \mathrm{~m}$ & $7.46 \times 10^{-10}( \pm 0.162)$ \\
\hline $6 n$ & $2.60 \times 10^{-10}( \pm 0.114)$ \\
\hline Nifed & $6.01 \times 10^{-10}( \pm 0.201)$ \\
\hline
\end{tabular}

a) $\mathrm{IC}_{50} \pm$ S.E.M. is determined graphically from dose-response curve in molar concentration and each compound is tested in triplicate.<smiles></smiles>

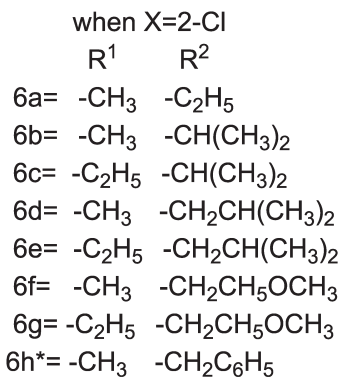

Fig. 1. Chemical Structures of the Synthesized 1,4-Dihydropyridines; Symmetrical Compounds (3a-k) and Asymmetrical Compounds (6a-n)

cates that the obtained results were due to the intrinsic activity of each compound. The synthesized compounds showed activity similar to, higher or lower than the reference compound; nifedipine. The most active compound was $\mathbf{3 h}$ which is three times more active than nifedipine.

Molecular Docking Molecular docking study was anticipated to interpret the comparative differences in the binding interactions of the synthesized compounds with marked activity at molecular level. This study was carried out on 3a and $\mathbf{f}-\mathbf{h}$ along with the reference molecule, nifedipine, into the DHPs receptor model active site using Molegro Virtual Docker (MVD) V6.0. The pharmacological activity results of the target compounds revealed that $\mathbf{3 f}$ and $\mathbf{h}$ were the least and the highest active calcium antagonists among this series, respectively. 3a and $\mathbf{g}$ have methyl ester group at 3- and 5-positions similar to nifedipine. This gives the opportunity to explore the effect of introducing the chlorophenyl ring at 4-position of the DHP ring. 


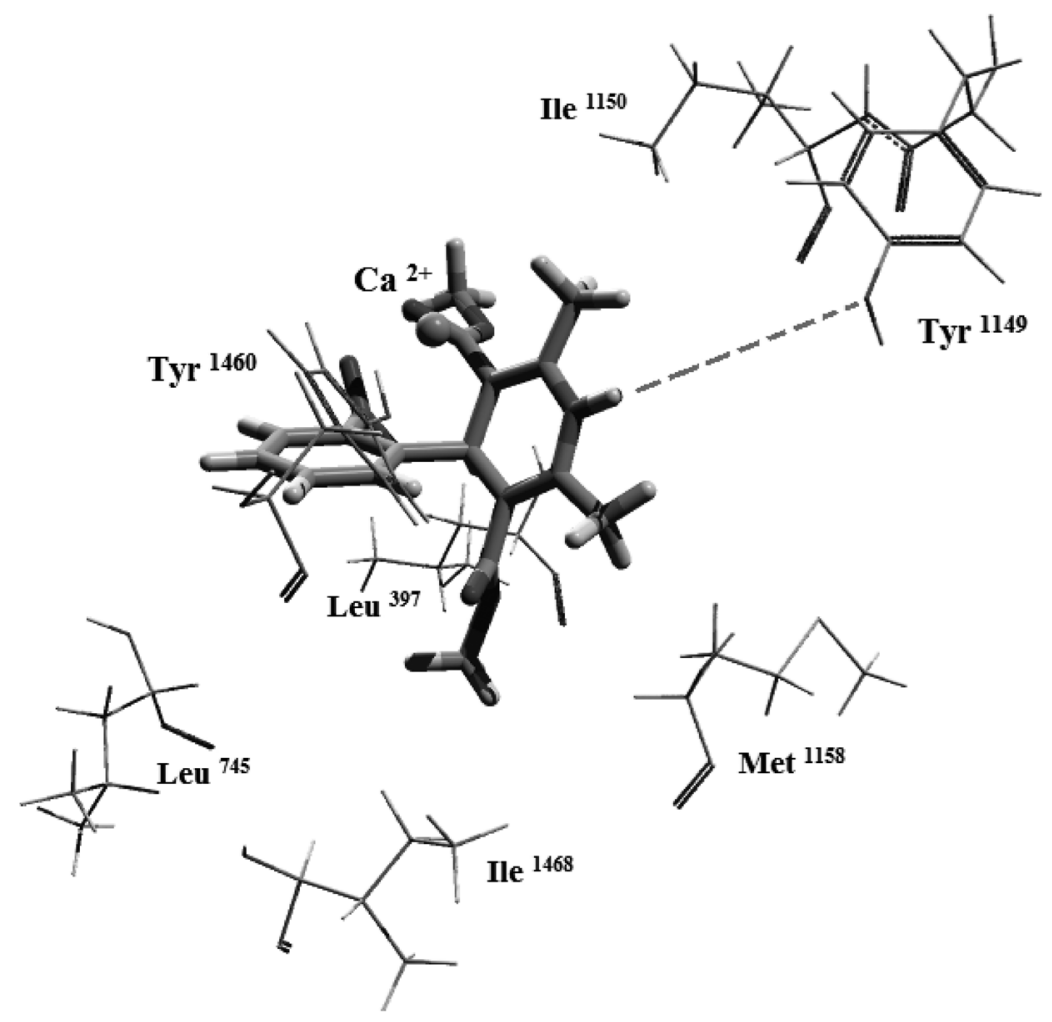

Fig. 2. Nifedipine Interaction with the DHP Receptor Model Showing H-Bond with Tyr $^{1149}$, Hydrophobic Attraction Force with Tyr ${ }^{1460}$, Chelate with $\mathrm{Ca}^{2+}$ Cofactor and Hydrophobic Interaction with the Lipophilic Bracelet $\left(\mathrm{Leu}^{397}, \mathrm{Leu}^{745}, \mathrm{Met}^{1158}\right.$ and Ile ${ }^{1468}$ ) and with $\mathrm{Ile}^{1150}$

Nifedipine affinity to the receptor can be explained by interaction with seven residues (Fig. 2). NH group of the DHP ring makes $\mathrm{H}$-bond with $\mathrm{Tyr}^{1149}$. Hydrophobic attraction force is observed between the phenyl ring of nifedipine and $\mathrm{Tyr}^{1460}$. The nitro group and oxygen of the carbonyl group in nifedipine chelate the $\mathrm{Ca}^{2+}$ cofactor of the selectivity filter. The down ester methyl group makes hydrophobic interaction with the lipophilic bracelet $\left(\mathrm{Leu}^{397}\right.$, Leu ${ }^{745}$, Met ${ }^{158}$ and $\left.\mathrm{Ile}^{1468}\right)$. The methyl group of DHP ring makes hydrophobic interaction with Ile ${ }^{1150}$.

Comparing the $\mathrm{IC}_{50}$ values of $\mathbf{3 a}$ and $\mathbf{g}$ with nifedipine, both are more active than nifedipine. This can be explained by the effect of introducing chlorophenyl ring in the synthesized compounds. Chlorine atom and nitro group are electron withdrawing groups however chlorine atom is lipophilic in nature in contrast to the hydrophilic nature of the nitro group. Chlorophenyl ring increases the lipophilicity of the synthesized compounds. Also ring to ring hydrophobic interaction between the substituted phenyl ring in nifedipine and the phenyl ring in $\mathrm{Tyr}^{1460}$ was preserved in the synthesized compounds. There is a non-significant difference in activity regarding to the position of chlorine atom on the phenyl ring which was obvious from the $\mathrm{IC}_{50}$ values of $\mathbf{3 a}$ and $\mathbf{g}\left(4.27 \times 10^{-10} \mathrm{M}\right.$ and $4.88 \times 10^{-10} \mathrm{M}$, respectively). This may indicate that substitution at ortho- or meta-positions makes the phenyl ring non coplanar with the DHP ring. Although nifedipine contains nitro group that included in the bidentate chelation of $\mathrm{Ca}^{2+}$ cofactor and absence of this property in the chlorine atom in the synthesized series, this series has higher calcium antagonist activity than nifedipine. This again implies the importance of the ring lipophilicity in the calcium antagonist activity of DHPs. ${ }^{33)}$ Ester groups at 3- and 5-positions are simply methyl ester groups in both nifedipine and the two compounds $\mathbf{3 a}$ and g. The down methyl ester group stabilizes the lipophilic bracelet while the other methyl ester group is projected in the water lake core of the receptor and participates in chelating the $\mathrm{Ca}^{2+}$ cofactor (Fig. 3). Hydrogen bonding between NH group of the DHP ring and hydroxyl group of $\mathrm{Tyr}^{1149}$ was observed in 3a and $\mathbf{g}$ interaction with the receptor.

Increasing lipophilicity is directly proportional to the calcium antagonist activity ${ }^{24)}$ however; steric hindrance is a limitation to this rule. When observing the $\mathrm{IC}_{50}$ values of this series, it is notable that this series cannot tolerate side chain more than total four carbon atoms (ethyl ester at 3- and 5-positions). $\mathbf{3 f}$ is the least active among the series. This compound has benzyl ester substituent with total ten carbon atom side chain (five extended carbon atom at 3- and 5-positions). This bulky side chain may interfere with the affinity axis ( $\operatorname{Tyr}^{1149}$ and $\mathrm{Tyr}^{1460}$ ) binding ${ }^{33)}$ which may result in abolishing ring to ring hydrophobic attraction force between the chlorophenyl ring and $\mathrm{Tyr}^{1460}$. The down benzyl ester group phenyl ring makes the affinity axis instead of important stabilization of the hydrophobic bracelet. This greatly decreases the activity of 3f regardless of increasing the lipophilicity of the compound (Fig. 4).

The highest active compound among the synthesized series was $\mathbf{3 h}$ which has the optimum side chain length. This compound is three times more active than nifedipine. The ethyl ester group makes the proper stabilization of the hydrophobic bracelet and minimal projection in the water lake. The phenyl ring in 4-position of DHP ring makes ring to ring hydrophobic attraction with $\mathrm{Tyr}^{1460}$. The chlorine atom in meta-position dose not interfere with this interaction, in contrast to orthoposition. The chlorine atom in ortho-position decreases to 

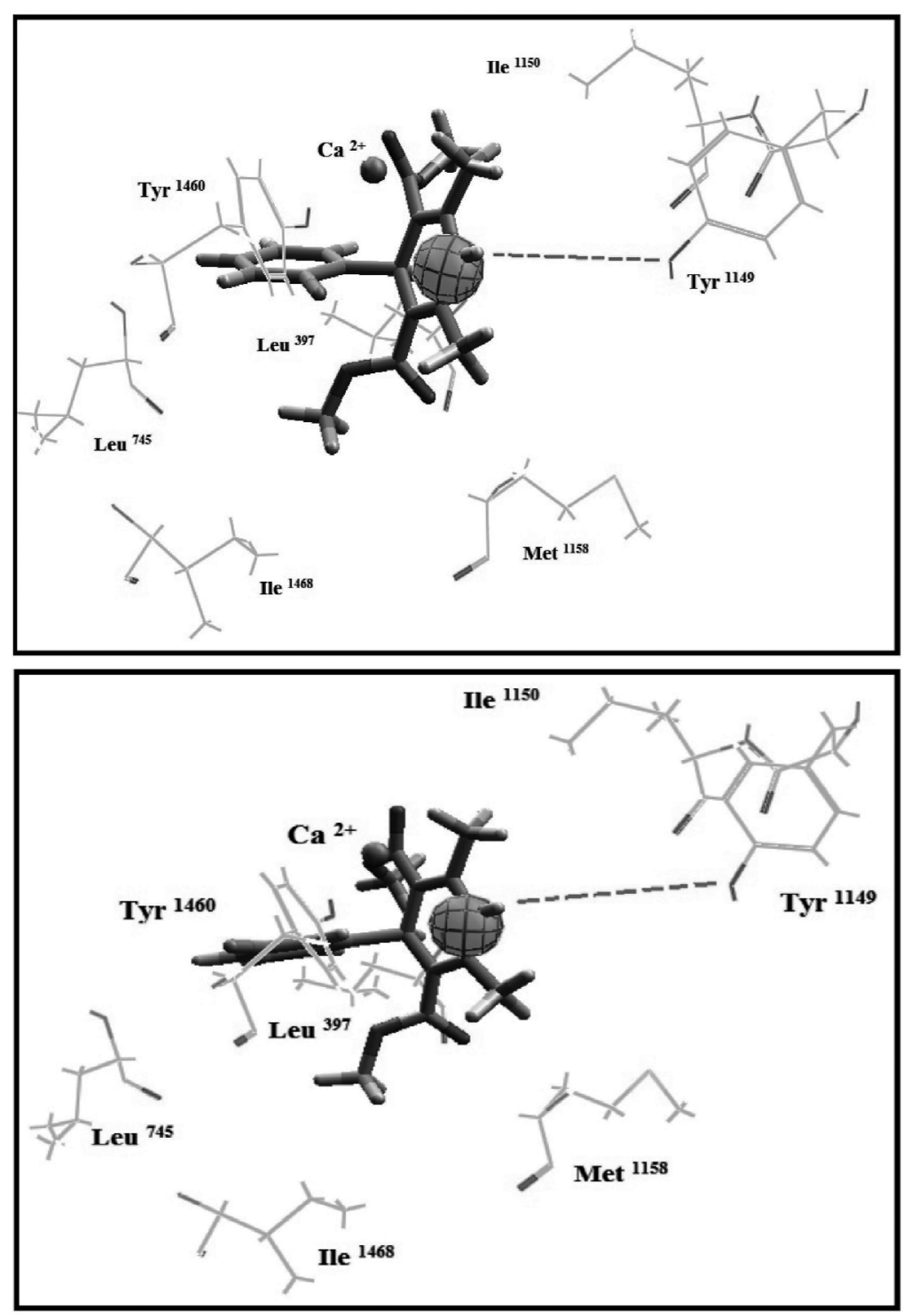

Fig. 3. Molecular Docking of 3a (Top) and 3g (Bottom) into the Active Site of DHP Receptor Model Showing Interaction Similar to Nifedipine (HBond with Tyr ${ }^{1149}$, Hydrophobic Attraction Force with $\mathrm{Tyr}^{1460}$, Chelate with $\mathrm{Ca}^{2+}$ Cofactor and Hydrophobic Interaction with the Lipophilic Bracelet and with Ile ${ }^{1150}$ )

some extent ring to ring hydrophobic attraction force due to clash with the $\mathrm{Ca}^{2+}$ cofactor of the selectivity filter (Fig. 5).

\section{Experimental}

Chemistry Melting points were determined on a Stuart SMP10 capillary melting point apparatus and are uncorrected. Elemental analyses were performed by Micro Analytical Center, Faculty of Science, Cairo University, Giza, Egypt and were within $\pm 0.4 \%$ of the calculated values. ${ }^{1} \mathrm{H}-\mathrm{NMR}$ spectra were recorded on a JEOL-ECA500 $(500 \mathrm{MHz})$ spectrometer. Samples were dissolved in $\mathrm{CDCl}_{3}$. Coupling constants are reported in $\mathrm{Hz}$. All organic reagents used were obtained from Sigma-Aldrich Company and were used without further purification. Reactions were monitored by TLC using pre-coated sheet (Fastman Kodak Co., Silca $60 \mathrm{~F}_{254}$ ) and were visualized with UV light at $254 \mathrm{~nm}$. Oily products were purified with preparative TLC (Silca gel) using chloroform as eluent.

General Procedure for the Synthesis of Symmetrical
Esters of Dialkyl 4-(Chlorophenyl)-2,6-dimethyl-1,4-dihydropyridine-3,5-dicarboxylate (3a-k) To $50 \mathrm{~mL}$ roundbottomed flask, ammonium acetate $(0.162 \mathrm{~g}, 2.10 \mathrm{mmol})$ was added to a stirring solution of 2-chlorobenzaldehyde or 3-chlorobenzaldehyde $(0.254 \mathrm{~g}, 1.81 \mathrm{mmol})$ and the corresponding alkyl acetoacetate $(3.62 \mathrm{mmol})$ in $10 \mathrm{~mL}$ methanol or 2-propanol. The reaction mixture was protected from light and heated under reflux for $12-24 \mathrm{~h}$. After cooling, the precipitate was filtered and purified by crystallization from methanol or 2-propanol to afford the corresponding product.

Dimethyl 4-(2-Chlorophenyl)-2,6-dimethyl-1,4-dihydropyridine3,5-dicarboxylate (3a)

Recrystallization from methanol afforded $0.480 \mathrm{~g}(79 \%)$ of the desired compound, mp: $186-188^{\circ} \mathrm{C}$ (lit. $\mathrm{mp} 186-188^{\circ} \mathrm{C}^{34)}$ ).

Diethyl 4-(2-Chlorophenyl)-2,6-dimethyl-1,4-dihydropyridine3,5-dicarboxylate $\mathbf{( 3 b )}$

Recrystallization from methanol afforded $0.434 \mathrm{~g}(66 \%)$ of the desired compound, mp: $128-130^{\circ} \mathrm{C}$ (lit. mp $128-130^{\circ} \mathrm{C}^{34)}$ ). 


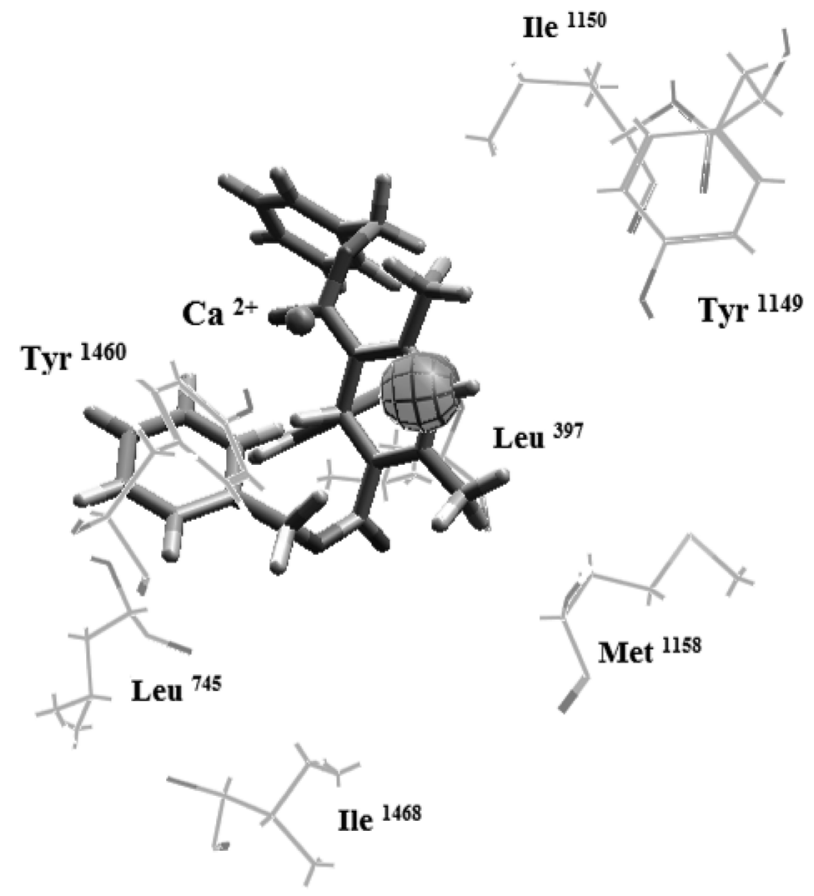

Fig. 4. Molecular Docking of $\mathbf{3 f}$ into the Active Site of DHP Receptor Model Showing That the Bulky Benzyl Ester Group Makes the Affinity Axis (Tyr ${ }^{149}$ and Tyr $^{1460}$ ) Binding Instead of Important Stabilization of the Hydrophobic Bracelet

Diisopropyl 4-(2-Chlorophenyl)-2,6-dimethyl-1,4-dihydropyridine-3,5-dicarboxylate (3c)

Recrystallization from methanol afforded $0.355 \mathrm{~g}$ (50\%), mp: $132-134^{\circ} \mathrm{C}$, Anal. (\%): $\left(\mathrm{C}_{21} \mathrm{H}_{26} \mathrm{ClNO}_{4}\right)$, Calcd (Found), C: 64.36 (64.22), H: 6.69 (6.46), N: 3.57 (3.53). ${ }^{1} \mathrm{H}-\mathrm{NMR}$ $\left(500 \mathrm{MHz}, \mathrm{CDCl}_{3}\right) \delta: 1.05\left(\mathrm{~d}, J=5.9 \mathrm{~Hz}, 6 \mathrm{H}, \mathrm{CH}\left(\mathrm{CH}_{3}\right)_{2}\right), 1.22$ $\left(\mathrm{d}, J=5.9 \mathrm{~Hz}, 6 \mathrm{H}, \mathrm{CH}\left(\mathrm{CH}_{3}\right)_{2}\right), 2.28\left(\mathrm{~s}, 6 \mathrm{H}, \mathrm{C}_{2}-\mathrm{CH}_{3}\right.$ and $\mathrm{C}_{6}-$ $\left.\mathrm{CH}_{3}\right), 4.85-5.01\left(\mathrm{~m}, 2 \mathrm{H}, 2\right.$ of $\left.\underline{\mathrm{CH}}\left(\mathrm{CH}_{3}\right)_{2}\right), 5.36\left(\mathrm{~s}, 1 \mathrm{H}, \mathrm{C}_{4}-\mathrm{H}\right)$, $5.67(\mathrm{~s}, 1 \mathrm{H}, \mathrm{NH}), 7.02\left(\mathrm{t}, J=8.5 \mathrm{~Hz}, 1 \mathrm{H}\right.$, aromatic $\left.\mathrm{C}\left(5^{\prime}\right) \mathrm{H}\right), 7.11$ (t, $J=7.5 \mathrm{~Hz}, 1 \mathrm{H}$, aromatic $\left.\mathrm{C}\left(4^{\prime}\right) \mathrm{H}\right), 7.21(\mathrm{~d}, J=8.5 \mathrm{~Hz}, 1 \mathrm{H}$, aromatic $\left.\mathrm{C}\left(6^{\prime}\right) \mathrm{H}\right), 7.37\left(\mathrm{~d}, J=7.5 \mathrm{~Hz}, 1 \mathrm{H}\right.$, aromatic $\left.\mathrm{C}\left(3^{\prime}\right) \mathrm{H}\right)$.

Diisobutyl 4-(2-Chlorophenyl)-2,6-dimethyl-1,4-dihydropyridine-3,5-dicarboxylate (3d)

Recrystallization from methanol afforded $0.160 \mathrm{~g}(21 \%)$, mp: $135-137^{\circ} \mathrm{C}$, Anal. (\%): $\left(\mathrm{C}_{23} \mathrm{H}_{30} \mathrm{ClNO}_{4}\right)$, Calcd (Found), C: 65.78 (65.90), H: 7.20 (6.95), N: 3.34 (3.26). ${ }^{1} \mathrm{H}-\mathrm{NMR}$ $\left(500 \mathrm{MHz}, \mathrm{CDCl}_{3}\right) \delta: 0.80\left(\mathrm{~d}, J=5.8 \mathrm{~Hz}, 6 \mathrm{H}, \mathrm{CH}\left(\mathrm{CH}_{3}\right)_{2}\right)$, $0.86\left(\mathrm{~d}, J=5.8 \mathrm{~Hz}, 6 \mathrm{H}, \mathrm{CH}\left(\mathrm{CH}_{3}\right)_{2}\right), 1.88-2.01(\mathrm{~m}, 2 \mathrm{H}, 2$ of $\left.\mathrm{CH}\left(\mathrm{CH}_{3}\right)_{2}\right), 2.31\left(\mathrm{~s}, 6 \mathrm{H}, \mathrm{C}_{2}-\mathrm{CH}_{3}\right.$ and $\left.\mathrm{C}_{6}-\mathrm{CH}_{3}\right), 3.70-3.90(\mathrm{~m}$, $4 \mathrm{H}, 2$ of $\left.\mathrm{COOCH}_{2}\right), 4.50\left(\mathrm{~s}, 1 \mathrm{H}, \mathrm{C}_{4}-\mathrm{H}\right), 5.62(\mathrm{~s}, 1 \mathrm{H}, \mathrm{NH}), 7.02$ $\left(\mathrm{t}, J=8.5 \mathrm{~Hz}, 1 \mathrm{H}\right.$, aromatic $\left.\mathrm{C}\left(5^{\prime}\right) \mathrm{H}\right), 7.12(\mathrm{t}, J=7.5 \mathrm{~Hz}, 1 \mathrm{H}$, aromatic $\left.\mathrm{C}\left(4^{\prime}\right) \mathrm{H}\right), 7.22\left(\mathrm{~d}, J=8.5 \mathrm{~Hz}, 1 \mathrm{H}\right.$, aromatic $\left.\mathrm{C}\left(6^{\prime}\right) \mathrm{H}\right), 7.40$ $\left(\mathrm{d}, J=7.5 \mathrm{~Hz}, 1 \mathrm{H}\right.$, aromatic $\left.\mathrm{C}\left(3^{\prime}\right) \mathrm{H}\right)$.

Bis(2-methoxyethyl) 4-(2-Chlorophenyl)-2,6-dimethyl-1,4dihydropyridine-3,5-dicarboxylate (3e)

Recrystallization from methanol afforded $0.445 \mathrm{~g}$ (58\%), mp: $124-126^{\circ} \mathrm{C}$, Anal. (\%): $\left(\mathrm{C}_{21} \mathrm{H}_{26} \mathrm{ClNO}_{6}\right)$, Calcd (Found), $\mathrm{C}: 59.50$ (59.68), H: 6.18 (6.38), N: 3.30 (3.34). ${ }^{1} \mathrm{H}-\mathrm{NMR}$ $\left(500 \mathrm{MHz}, \mathrm{CDCl}_{3}\right) \delta: 2.27\left(\mathrm{~s}, 6 \mathrm{H}, \mathrm{C}_{2}-\underline{\mathrm{CH}}_{3}\right.$ and $\left.\mathrm{C}_{6}-\mathrm{CH}_{3}\right), 3.27$ (s, 6H, 2 of $\left.\mathrm{OCH}_{3}\right), 3.46-3.63\left(\mathrm{~m}, 4 \mathrm{H}, 2\right.$ of $\left.\mathrm{COOCH}_{2} \mathrm{CH}_{2} \mathrm{O}\right)$, 4.04-4.24 (m, 4H, 2 of $\left.\mathrm{COOCH}_{2}\right), 5.37\left(\mathrm{~s}, 1 \mathrm{H}, \mathrm{C}_{4}-\mathrm{H}\right), 5.65$ (s, 1H, NH), $7.03\left(\mathrm{t}, J=8.5 \mathrm{~Hz}, 1 \mathrm{H}\right.$, aromatic $\left.\mathrm{C}\left(5^{\prime}\right) \mathrm{H}\right), 7.11(\mathrm{t}$, $J=7.5 \mathrm{~Hz}, 1 \mathrm{H}$, aromatic $\left.\mathrm{C}\left(4^{\prime}\right) \mathrm{H}\right), 7.23(\mathrm{~d}, J=8.5 \mathrm{~Hz}, 1 \mathrm{H}$, aro-

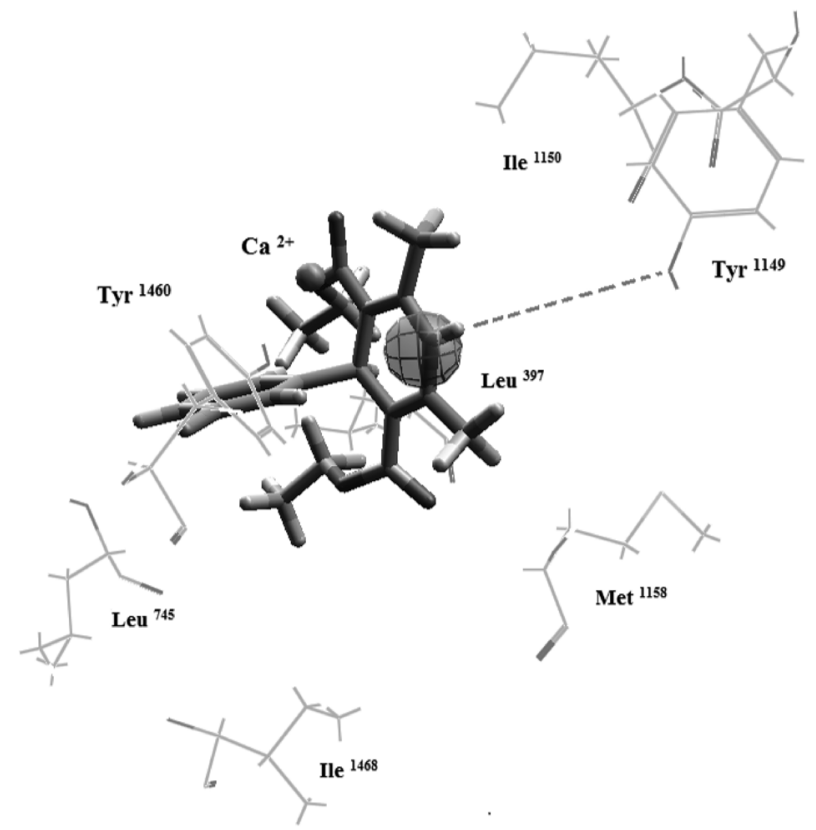

Fig. 5. Molecular Docking of $\mathbf{3 h}$ into the Active Site of DHP Receptor Model Showing Interaction Like Nifidepine (H-Bond with Tyr ${ }^{1149}$, Hydrophobic Attraction Force with $\mathrm{Tyr}^{1460}$, Chelate with $\mathrm{Ca}^{2+}$ Cofactor and Hydrophobic Interaction with the Lipophilic Bracelet and with $\mathrm{Ile}^{1150}$ ) and Proper Chlorine Atom Position Away from the Hydrophilic Core of the Receptor

matic $\left.\mathrm{C}\left(6^{\prime}\right) \mathrm{H}\right), 7.38\left(\mathrm{~d}, J=7.5 \mathrm{~Hz}, 1 \mathrm{H}\right.$, aromatic $\left.\mathrm{C}\left(3^{\prime}\right) \mathrm{H}\right)$.

Dibenzyl 4-(2-Chlorophenyl)-2,6-dimethyl-1,4-dihydropyridine-3,5-dicarboxylate (3f)

TLC purification afforded $0.706 \mathrm{~g}$ (80\%), pure oil. (\%): $\left(\mathrm{C}_{29} \mathrm{H}_{26} \mathrm{ClNO}_{4}\right)$, Calcd (Found), C: 71.38 (71.22), H: 5.37 (5.35), $\mathrm{N}: 2.87$ (2.79). ${ }^{1} \mathrm{H}-\mathrm{NMR}\left(500 \mathrm{MHz}, \mathrm{CDCl}_{3}\right) \delta: 2.25(\mathrm{~s}, 6 \mathrm{H}$, $\mathrm{C}_{2}-\mathrm{CH}_{3}$ and $\left.\mathrm{C}_{6}-\mathrm{CH}_{3}\right), 5.03\left(\mathrm{~d}, J=20.1 \mathrm{~Hz}, 2 \mathrm{H}, \mathrm{COOCH}_{2}\right), 5.06$ (d, $\left.J=21.3 \mathrm{~Hz}, 2 \mathrm{H}, \mathrm{COOCH}_{2}\right), 5.42\left(\mathrm{~s}, 1 \mathrm{H}, \mathrm{C}_{4}-\mathrm{H}\right), 5.80(\mathrm{~s}, 1 \mathrm{H}$, $\mathrm{NH}), 7.01-7.07$ (m, 2H, aromatic $\mathrm{C}\left(5^{\prime}\right) \mathrm{H}$ and $\left.\mathrm{C}\left(4^{\prime}\right) \mathrm{H}\right), 7.13-7.31$ (m, 12H, aromatic $\mathrm{OCH}_{2} \underline{\mathrm{C}}_{6} \underline{\mathrm{H}}_{5}, \mathrm{C}\left(6^{\prime}\right) \mathrm{H}$ and $\left.\mathrm{C}\left(3^{\prime}\right) \mathrm{H}\right)$.

Dimethyl 4-(3-Chlorophenyl)-2,6-dimethyl-1,4-dihydropyridine-3,5-dicarboxylate $\mathbf{( 3 g})$

Recrystallization from methanol afforded $0.201 \mathrm{~g}(60 \%$ yield) of the desired compound, mp: $188-190^{\circ} \mathrm{C}$ (lit. $\mathrm{mp}$ $\left.188-190^{\circ} \mathrm{C}^{34)}\right)$.

Diethyl 4-(3-Chlorophenyl)-2,6-dimethyl-1,4-dihydropyridine-3,5-dicarboxylate $\mathbf{( 3 h )}$

Recrystallization from methanol afforded $0.196 \mathrm{~g}$ (54\% yield) of the desired compound, mp: $132-134^{\circ} \mathrm{C}$ (lit. $\mathrm{mp}$ $\left.132-134^{\circ} \mathrm{C}^{34)}\right)$.

Diisoproyl 4-(3-Chlorophenyl)-2,6-dimethyl-1,4-dihydropyridine-3,5-dicarboxylate (3i)

Recrystallization from methanol afforded $0.567 \mathrm{~g}(80 \%)$, mp: $116-118^{\circ} \mathrm{C}$, Anal. (\%): $\left(\mathrm{C}_{21} \mathrm{H}_{26} \mathrm{ClNO}_{4}\right)$, Calcd (Found), C: 64.36 (64.09), H: 6.69 (6.39), N: 3.57 (3.58). ${ }^{1} \mathrm{H}-\mathrm{NMR}$ $\left(500 \mathrm{MHz}, \mathrm{CDCl}_{3}\right) \delta: 1.08\left(\mathrm{~d}, J=5.9 \mathrm{~Hz}, 6 \mathrm{H}, \mathrm{CH}\left(\underline{\mathrm{CH}}_{3}\right)_{2}\right), 1.20$ (d, $\left.J=5.9 \mathrm{~Hz}, 6 \mathrm{H}, \mathrm{CH}\left(\underline{\mathrm{CH}}_{3}\right)_{2}\right), 2.23\left(\mathrm{~s}, 6 \mathrm{H}, \mathrm{C}_{2}-\underline{\mathrm{CH}}_{3}\right.$ and $\mathrm{C}_{6}-$ $\left.\mathrm{CH}_{3}\right), 4.87-4.95\left(\mathrm{~m}, 2 \mathrm{H}, 2\right.$ of $\left.\underline{\mathrm{CH}}\left(\mathrm{CH}_{3}\right)_{2}\right), 5.58\left(\mathrm{~s}, 1 \mathrm{H}, \mathrm{C}_{4}-\mathrm{H}\right)$, $7.06-7.23\left(\mathrm{~m}, 3 \mathrm{H}\right.$, aromatic $\mathrm{C}\left(\overline{\left.6^{\prime}\right) \mathrm{H}}, \mathrm{C}\left(5^{\prime}\right) \mathrm{H}\right.$ and $\left.\mathrm{C}\left(4^{\prime}\right) \mathrm{H}\right), 7.27$ (s, $1 \mathrm{H}$, aromatic $\left.\mathrm{C}\left(2^{\prime}\right) \mathrm{H}\right)$.

Diisobutyl 4-(3-Chlorophenyl)-2,6-dimethyl-1,4-dihydropyridine-3,5-dicarboxylate $(\mathbf{3 j})$

Recrystallization from methanol afforded $0.175 \mathrm{~g}$ (23\%), mp: 
95-97 ${ }^{\circ} \mathrm{C}$, Anal. (\%): $\left(\mathrm{C}_{23} \mathrm{H}_{30} \mathrm{ClNO}_{4}\right)$, Calcd (Found), C: 65.78 (65.55), H: 7.20 (6.94), N: 3.34 (3.33). ${ }^{1} \mathrm{H}-\mathrm{NMR}(500 \mathrm{MHz}$, $\left.\mathrm{CDCl}_{3}\right) \quad \delta: 0.87\left(\mathrm{~d}, J=5.8 \mathrm{~Hz}, 6 \mathrm{H}, \quad \mathrm{CH}\left(\underline{\mathrm{CH}}_{3}\right)_{2}\right), 0.90$ (d, $\left.J=5.8 \mathrm{~Hz}, 6 \mathrm{H}, \mathrm{CH}\left(\mathrm{CH}_{3}\right)_{2}\right), 1.80-1.96\left(\mathrm{~m}, 2 \mathrm{H}, 2\right.$ of $\left.\underline{\mathrm{CH}}\left(\mathrm{CH}_{3}\right)_{2}\right)$, $2.32\left(\mathrm{~s}, 6 \mathrm{H}, \mathrm{C}_{2}-\mathrm{CH}_{3}\right.$ and $\left.\mathrm{C}_{6}-\mathrm{CH}_{3}\right), 3.83(\mathrm{~d}, J=6.0 \mathrm{~Hz}, 4 \mathrm{H}, 2$ of $\left.\mathrm{COOCH}_{2}\right), 4.89\left(\mathrm{~s}, 1 \mathrm{H}, \mathrm{C}_{4}-\mathrm{H}\right), 5.79(\mathrm{~s}, 1 \mathrm{H}, \mathrm{NH}), 6.99-7.21(\mathrm{~m}$, $3 \mathrm{H}$, aromatic $\mathrm{C}\left(6^{\prime}\right) \mathrm{H}, \mathrm{C}\left(5^{\prime}\right) \mathrm{H}$ and $\left.\mathrm{C}\left(4^{\prime}\right) \mathrm{H}\right), 7.28$ (s, 1H, aromatic $\left.\mathrm{C}\left(2^{\prime}\right) \mathrm{H}\right)$.

Bis(2-methoxyethyl) 4-(3-Chlorophenyl)-2,6-dimethyl-1,4dihydropyridine-3,5-dicarboxylate $(\mathbf{3 k})$

Recrystallization from methanol afforded $0.476 \mathrm{~g}(62 \%), \mathrm{mp}$ : 118-120 ${ }^{\circ} \mathrm{C}$, Anal. (\%): $\left(\mathrm{C}_{21} \mathrm{H}_{26} \mathrm{ClNO}_{6}\right)$, Calcd (Found), C: 59.50 (59.19), H: 6.18 (5.92), N: 3.30 (3.28). ${ }^{1} \mathrm{H}-\mathrm{NMR}(500 \mathrm{MHz}$, $\left.\mathrm{CDCl}_{3}\right) \delta: 2.31\left(\mathrm{~s}, 6 \mathrm{H}, \mathrm{C}_{2}-\mathrm{CH}_{3}\right.$ and $\left.\mathrm{C}_{6}-\mathrm{CH}_{3}\right), 3.33(\mathrm{~s}, 6 \mathrm{H}, 2$ of $\left.\mathrm{OCH}_{3}\right), 3.49-3.62\left(\mathrm{~m}, 4 \mathrm{H}, 2\right.$ of $\left.\mathrm{COOCH}_{2} \mathrm{CH}_{2} \mathrm{O}\right), 4.06-4.24$ $\left(\mathrm{m}, \overline{\mathrm{H}}, 2\right.$ of $\left.\mathrm{COOCH}_{2}\right), 4.96\left(\mathrm{~s}, 1 \mathrm{H}, \mathrm{C}_{4}-\mathrm{H}\right), 6.99-7.15(\mathrm{~m}, 2 \mathrm{H}$, aromatic $\mathrm{C}\left(6^{\prime}\right) \mathrm{H}$ and $\left.\mathrm{C}\left(5^{\prime}\right) \mathrm{H}\right) ; 7.19(\mathrm{~d}, J=8.8 \mathrm{~Hz}, 1 \mathrm{H}$, aromatic $\left.\mathrm{C}\left(4^{\prime}\right) \mathrm{H}\right) ; 7.26\left(\mathrm{~s}, 1 \mathrm{H}\right.$, aromatic $\left.\mathrm{C}\left(2^{\prime}\right) \mathrm{H}\right)$.

General Procedure for the Synthesis of Asymmetrical Esters of Dialkyl 4-(Chlorophenyl)-2,6-dimethyl-1,4-dihydropyridine-3,5-dicarboxylate $(\mathbf{6 a}-\mathbf{n})$ To a $50 \mathrm{~mL}$ roundbottomed flask were added a mixture of 2-chlorobenzaldehyde or 3-chlorobenzaldehyde $(0.250 \mathrm{~g}, 1.78 \mathrm{mmol})$, alkyl acetoacetate $(1.78 \mathrm{mmol})$ and alkyl 3 -aminocrotonate $(1.78 \mathrm{mmol})$ in $10 \mathrm{~mL}$ methanol or 2-propanol. The reaction mixture was protected from light and heated under reflux for 9-24h. After cooling, the precipitate was filtered and purified by crystallization from methanol or 2-propanol to afford the corresponding product.

5-Ethyl 3-Methyl 4-(2-Chlorophenyl)-2,6-dimethyl-1,4dihydropyridine-3,5-dicarboxylate (6a)

Recrystallization from methanol afforded $0.189 \mathrm{~g} \mathrm{(30 \% ),}$ mp: $113-115^{\circ} \mathrm{C}$, Anal. (\%): $\left(\mathrm{C}_{18} \mathrm{H}_{20} \mathrm{ClNO}_{4}\right)$, Calcd (Found), $\mathrm{C}: 61.80$ (62.09), H: 5.76 (5.50), N: 4.00 (4.18). ${ }^{1} \mathrm{H}-\mathrm{NMR}$ $\left(500 \mathrm{MHz}, \mathrm{CDCl}_{3}\right) \delta: 1.17\left(\mathrm{t}, J=6.1 \mathrm{~Hz}, 3 \mathrm{H}, \mathrm{CH}_{2} \mathrm{CH}_{3}\right), 2.25$ (s, $6 \mathrm{H}, \mathrm{C}_{2}-\mathrm{CH}_{3}$ and $\left.\mathrm{C}_{6}-\underline{\mathrm{CH}}_{3}\right), 3.59\left(\mathrm{~s}, 3 \mathrm{H}, \mathrm{COOC} \underline{\mathrm{CH}}_{3}\right), 3.98-4.14$ $\left(\mathrm{m}, 2 \mathrm{H}, \mathrm{COOCH}_{2}\right), 5.40\left(\mathrm{~s}, 1 \mathrm{H}, \mathrm{C}_{4}-\mathrm{H}\right), 5.69(\mathrm{~s}, 1 \mathrm{H}, \mathrm{NH}), 7.01$ (t, $J=8.5 \mathrm{~Hz}, 1 \mathrm{H}$, aromatic $\left.\mathrm{C}\left(5^{\prime}\right) \mathrm{H}\right), 7.13$ (t, $J=7.5 \mathrm{~Hz}, 1 \mathrm{H}$, aromatic $\left.\mathrm{C}\left(4^{\prime}\right) \mathrm{H}\right), 7.22\left(\mathrm{~d}, J=8.5 \mathrm{~Hz}, 1 \mathrm{H}\right.$, aromatic $\left.\mathrm{C}\left(6^{\prime}\right) \mathrm{H}\right), 7.37$ $\left(\mathrm{d}, J=7.5 \mathrm{~Hz}, 1 \mathrm{H}\right.$, aromatic $\left.\mathrm{C}\left(3^{\prime}\right) \mathrm{H}\right)$.

5-Isopropyl 3-Methyl 4-(2-Chlorophenyl)-2,6-dimethyl-1,4dihydropyridine-3,5-dicarboxylate $(\mathbf{6 b})$

Recrystallization from methanol afforded $0.166 \mathrm{~g}$ (25\%), mp: 151-153 ${ }^{\circ} \mathrm{C}$, Anal. (\%): $\left(\mathrm{C}_{19} \mathrm{H}_{22} \mathrm{ClNO}_{4}\right)$, Calcd (Found), C: 62.72 (62.42), H: 6.09 (5.84), N: 3.85 (3.89). ${ }^{1} \mathrm{H}-\mathrm{NMR}(500 \mathrm{MHz}$, $\left.\mathrm{CDCl}_{3}\right) \delta: 1.00\left(\mathrm{~d}, J=5.9 \mathrm{~Hz}, 3 \mathrm{H}, \mathrm{CH}\left(\mathrm{CH}_{3}\right)_{2}\right), 1.19(\mathrm{~d}, J=5.9 \mathrm{~Hz}$, $\left.3 \mathrm{H}, \mathrm{CH}\left(\mathrm{CH}_{3}\right)_{2}\right), 2.22\left(\mathrm{~s}, 3 \mathrm{H}, \mathrm{C}_{2}-\mathrm{CH}_{3}\right), 2.32\left(\mathrm{~s}, 3 \mathrm{H}, \mathrm{C}_{6}-\mathrm{CH}_{3}\right)$, $3.55\left(\mathrm{~s}, 3 \mathrm{H}, \mathrm{COOCH}_{3}\right), 4.85-5.05\left(\mathrm{~m}, 1 \mathrm{H}, \mathrm{CH}\left(\mathrm{CH}_{3}\right)_{2}\right), 5.31$ $\left(\mathrm{s}, 1 \mathrm{H}, \mathrm{C}_{4}-\mathrm{H}\right), 5.71(\mathrm{~s}, 1 \mathrm{H}, \mathrm{NH}), 7.00(\mathrm{t}, J=8.5 \mathrm{~Hz}, 1 \mathrm{H}$, aromatic $\left.\mathrm{C}\left(5^{\prime}\right) \mathrm{H}\right), 7.11\left(\mathrm{t}, J=7.5 \mathrm{~Hz}, 1 \mathrm{H}\right.$, aromatic $\left.\mathrm{C}\left(4^{\prime}\right) \mathrm{H}\right), 7.21$ (d, $J=8.5 \mathrm{~Hz}, 1 \mathrm{H}$, aromatic $\left.\mathrm{C}\left(6^{\prime}\right) \mathrm{H}\right), 7.35(\mathrm{~d}, J=7.5 \mathrm{~Hz}, 1 \mathrm{H}$, aromatic $\left.\mathrm{C}\left(3^{\prime}\right) \mathrm{H}\right)$.

3-Ethyl 5-Isopropyl 4-(2-Chlorophenyl)-2,6-dimethyl-1,4dihydropyridine-3,5-dicarboxylate (6c)

Recrystallization from methanol afforded $0.483 \mathrm{~g}$ (71\%), mp: 115-117 ${ }^{\circ} \mathrm{C}$, Anal. (\%): $\left(\mathrm{C}_{20} \mathrm{H}_{24} \mathrm{ClNO}_{4}\right)$, Calcd (Found), C: 63.57 (63.38), H: 6.40 (6.09), N: 3.71 (3.57). ${ }^{1} \mathrm{H}-\mathrm{NMR}(500 \mathrm{MHz}$, $\left.\mathrm{CDCl}_{3}\right) \delta: 1.44\left(\mathrm{t}, J=6.1 \mathrm{~Hz}, 3 \mathrm{H}, \mathrm{CH}_{2} \mathrm{CH}_{3}\right), 1.18(\mathrm{~d}, J=6.0 \mathrm{~Hz}$, $\left.3 \mathrm{H}, \mathrm{CH}\left(\mathrm{CH}_{3}\right)_{2}\right), 1.24\left(\mathrm{~d}, J=6.0 \mathrm{~Hz}, 3 \mathrm{H}, \mathrm{CH}\left(\mathrm{CH}_{3}\right)_{2}\right), 2.21(\mathrm{~s}, 6 \mathrm{H}$, $\mathrm{C}_{2}-\mathrm{CH}_{3}$ and $\left.\mathrm{C}_{6}-\underline{\mathrm{CH}}_{3}\right), 3.82-4.20\left(\mathrm{~m}, 2 \mathrm{H}, \mathrm{COOCH}_{2}\right), 4.70-5.01$ $\left(\mathrm{m}, 1 \mathrm{H}, \underline{\mathrm{CH}}\left(\mathrm{CH}_{3}\right)_{2}\right), 5.28\left(\mathrm{~s}, 1 \mathrm{H}, \mathrm{C}_{4}-\mathrm{H}\right), 5.72(\mathrm{~s}, 1 \mathrm{H}, \mathrm{NH}), 6.98$ (t, $J=8.5 \mathrm{~Hz}, 1 \mathrm{H}$, aromatic $\left.\mathrm{C}\left(5^{\prime}\right) \mathrm{H}\right), 7.11(\mathrm{t}, J=7.5 \mathrm{~Hz}, 1 \mathrm{H}$, aromatic $\left.\mathrm{C}\left(4^{\prime}\right) \mathrm{H}\right), 7.21\left(\mathrm{~d}, J=8.5 \mathrm{~Hz}, 1 \mathrm{H}\right.$, aromatic $\left.\mathrm{C}\left(6^{\prime}\right) \mathrm{H}\right), 7.38$ $\left(\mathrm{d}, J=7.5 \mathrm{~Hz}\right.$, aromatic $\left.1 \mathrm{H}, \mathrm{C}\left(3^{\prime}\right) \mathrm{H}\right)$.

5-Isobutyl 3-Methyl 4-(2-Chlorophenyl)-2,6-dimethyl-1,4dihydropyridine-3,5-dicarboxylate (6d)

Recrystallization from methanol afforded $0.215 \mathrm{~g} \mathrm{(32 \% ),} \mathrm{mp:}$ 158-160 ${ }^{\circ} \mathrm{C}$, Anal. (\%): $\left(\mathrm{C}_{20} \mathrm{H}_{24} \mathrm{ClNO}_{4}\right)$, Calcd (Found), C: 63.57 (63.40), H: 6.40 (6.15), N: 3.71 (3.57). ${ }^{1} \mathrm{H}-\mathrm{NMR}(500 \mathrm{MHz}$, $\left.\mathrm{CDCl}_{3}\right) \delta: 0.78\left(\mathrm{~d}, J=6.4 \mathrm{~Hz}, 6 \mathrm{H}, \mathrm{CH}\left(\mathrm{CH}_{3}\right)_{2}\right), 1.88-1.99(\mathrm{~m}$, $\left.1 \mathrm{H}, \underline{\mathrm{CH}}\left(\mathrm{CH}_{3}\right)_{2}\right), 2.28\left(\mathrm{~s}, 6 \mathrm{H}, \mathrm{C}_{2}-\mathrm{CH}_{3}\right.$ and $\left.\mathrm{C}_{6}-\underline{\mathrm{CH}}_{3}\right), 3.58(\mathrm{~s}$, $\left.3 \mathrm{H}, \mathrm{COOCH}_{3}\right), 3.84\left(\mathrm{~d}, J=7.3 \mathrm{~Hz}, 2 \mathrm{H}, \mathrm{COOCH}{ }_{2} \mathrm{CH}\right), 5.30$ $\left(\mathrm{s}, 1 \mathrm{H}, \mathrm{C}_{4}-\mathrm{H}\right), 5.68(\mathrm{~s}, 1 \mathrm{H}, \mathrm{NH}), 6.97(\mathrm{t}, J=8.5 \mathrm{~Hz}, 1 \mathrm{H}$, aromatic $\left.\mathrm{C}\left(5^{\prime}\right) \mathrm{H}\right), 7.11\left(\mathrm{t}, J=7.5 \mathrm{~Hz}, 1 \mathrm{H}\right.$, aromatic $\left.\mathrm{C}\left(4^{\prime}\right) \mathrm{H}\right), 7.23$ $\left(\mathrm{d}, J=8.5 \mathrm{~Hz}, 1 \mathrm{H}\right.$, aromatic $\left.\mathrm{C}\left(6^{\prime}\right) \mathrm{H}\right), 7.38(\mathrm{~d}, J=7.5 \mathrm{~Hz}, 1 \mathrm{H}$, aromatic $\left.\mathrm{C}\left(3^{\prime}\right) \mathrm{H}\right)$.

3-Ethyl 5-Isobutyl 4-(2-Chlorophenyl)-2,6-dimethyl-1,4dihydropyridine-3,5-dicarboxylate (6e)

Recrystallization from methanol afforded $0.196 \mathrm{~g}$ (28\%), mp: 114-116 ${ }^{\circ} \mathrm{C}$, Anal. (\%): $\left(\mathrm{C}_{21} \mathrm{H}_{26} \mathrm{ClNO}_{4}\right)$, Calcd (Found), C: 64.36 (64. 09), H: 6.69 (6.39), N: 3.57 (3.59). ${ }^{1} \mathrm{H}-\mathrm{NMR}(500 \mathrm{MHz}$, $\left.\mathrm{CDCl}_{3}\right) \delta: 0.74\left(\mathrm{~d}, J=6.5 \mathrm{~Hz}, 6 \mathrm{H}, \mathrm{CH}\left(\mathrm{CH}_{3}\right)_{2}\right), 1.15(\mathrm{t}, J=6.1 \mathrm{~Hz}$, $\left.3 \mathrm{H}, \mathrm{CH}_{2} \mathrm{CH}_{3}\right), 1.84-1.99\left(\mathrm{~m}, 1 \mathrm{H}, \underline{\mathrm{CH}}\left(\mathrm{CH}_{3}\right)_{2}\right), 2.27(\mathrm{~s}, 6 \mathrm{H}$, $\mathrm{C}_{2}-\underline{\mathrm{CH}}_{3}$ and $\left.\mathrm{C}_{6}-\underline{\mathrm{CH}}_{3}\right), 3.75\left(\mathrm{~d}, J=7.1 \mathrm{~Hz}, 2 \mathrm{H}, \mathrm{COOCH}_{2} \mathrm{CH}\right)$, $3.96-4.21\left(\mathrm{~m}, 2 \mathrm{H}, \mathrm{COOCH}_{2} \mathrm{CH}_{3}\right), 5.34\left(\mathrm{~s}, 1 \mathrm{H}, \mathrm{C}_{4}-\mathrm{H}\right), 5.77$ (s, $1 \mathrm{H}, \mathrm{NH}), 6.98\left(\mathrm{t}, J=8.5 \mathrm{~Hz}, 1 \mathrm{H}\right.$, aromatic $\left.\mathrm{C}\left(5^{\prime}\right) \mathrm{H}\right), 7.11(\mathrm{t}$, $J=7.5 \mathrm{~Hz}, 1 \mathrm{H}$, aromatic $\left.\mathrm{C}\left(4^{\prime}\right) \mathrm{H}\right), 7.23(\mathrm{~d}, J=8.5 \mathrm{~Hz}, 1 \mathrm{H}$, aromatic $\left.\mathrm{C}\left(6^{\prime}\right) \mathrm{H}\right), 7.37\left(\mathrm{~d}, J=7.5 \mathrm{~Hz}, 1 \mathrm{H}\right.$, aromatic $\left.\mathrm{C}\left(3^{\prime}\right) \mathrm{H}\right)$.

3-Methyl 5-(2-Methoxyethyl) 4-(2-Chlorophenyl)-2,6dimethyl-1,4-dihydropyridine-3,5-dicarboxylate (6f)

Recrystallization from methanol afforded $0.347 \mathrm{~g}(51 \%)$, mp: $103-105^{\circ} \mathrm{C}$, Anal. (\%): $\left(\mathrm{C}_{19} \mathrm{H}_{22} \mathrm{ClNO}_{5}\right)$, Calcd (Found), $\mathrm{C}: 60.08$ (59.76), H: 5.84 (5.61), N: 3.69 (3.44). ${ }^{1} \mathrm{H}-\mathrm{NMR}$ $\left(500 \mathrm{MHz}, \mathrm{CDCl}_{3}\right) \delta: 2.26\left(\mathrm{~s}, 6 \mathrm{H}, \mathrm{C}_{2}-\mathrm{CH}_{3}\right.$ and $\left.\mathrm{C}_{6}-\mathrm{CH}_{3}\right), 3.26$ (s, $\left.3 \mathrm{H}, \mathrm{OCH}_{3}\right), 3.52-3.58\left(\mathrm{~m}, 2 \mathrm{H}, \mathrm{COOCH}_{2} \mathrm{CH}_{2} \mathrm{O}\right), 3.66$ (s, $\left.3 \mathrm{H}, \mathrm{COOCH}_{3}\right), 4.05-4.26\left(\mathrm{~m}, 2 \mathrm{H}, \mathrm{COOCH}_{2} \mathrm{CH}_{2} \mathrm{O}\right), 5.42$ (s, $\left.1 \mathrm{H}, \mathrm{C}_{4}-\mathrm{H}\right), 5.77(\mathrm{~s}, 1 \mathrm{H}, \mathrm{NH}), 7.02(\mathrm{t}, J=8.5 \mathrm{~Hz}, 1 \mathrm{H}$, aromatic $\left.\mathrm{C}\left(5^{\prime}\right) \mathrm{H}\right), 7.11\left(\mathrm{t}, J=7.5 \mathrm{~Hz}, 1 \mathrm{H}\right.$, aromatic $\left.\mathrm{C}\left(4^{\prime}\right) \mathrm{H}\right), 7.22(\mathrm{~d}$, $J=8.5 \mathrm{~Hz}, 1 \mathrm{H}$, aromatic $\left.\mathrm{C}\left(6^{\prime}\right) \mathrm{H}\right), 7.36(\mathrm{~d}, J=7.5 \mathrm{~Hz}, 1 \mathrm{H}$, aromatic $\left.\mathrm{C}\left(3^{\prime}\right) \mathrm{H}\right)$.

3-Ethyl 5-(2-Methoxyethyl) 4-(2-Chlorophenyl)-2,6-dimethyl-1,4-dihydropyridine-3,5-dicarboxylate $(\mathbf{6 g})$

Recrystallization from methanol afforded $0.394 \mathrm{~g} \mathrm{(56 \% ),} \mathrm{mp:}$ 70-72 ${ }^{\circ} \mathrm{C}$, Anal. (\%): $\left(\mathrm{C}_{20} \mathrm{H}_{24} \mathrm{ClNO}_{5}\right)$, Calcd (Found), C: 60.99 (61.31), H: 6.14 (5.80), N: 3.56 (3.53). ${ }^{1} \mathrm{H}-\mathrm{NMR}(500 \mathrm{MHz}$, $\left.\mathrm{CDCl}_{3}\right) \delta: 1.17\left(\mathrm{t}, J=6.1 \mathrm{~Hz}, 3 \mathrm{H}, \mathrm{CH}_{2} \mathrm{CH}_{3}\right), 2.30\left(\mathrm{~s}, 6 \mathrm{H}, \mathrm{C}_{2}-\right.$ $\underline{\mathrm{CH}}_{3}$ and $\left.\mathrm{C}_{6}-\underline{\mathrm{CH}}_{3}\right), 3.29\left(\mathrm{~s}, 3 \mathrm{H}, \mathrm{OCH}_{3}\right), 3.44(\mathrm{t}, J=5.3 \mathrm{~Hz}, 2 \mathrm{H}$, $\mathrm{COOCH}_{2} \mathrm{CH}_{2} \mathrm{O}$ ), 3.51-3.61 (m, 2 $\left.\mathrm{H}, \mathrm{COOCH}_{2} \mathrm{CH}_{3}\right), 4.00-4.10$ $\left(\mathrm{m}, 2 \mathrm{H}, \mathrm{COOCH}_{2} \mathrm{CH}_{2} \mathrm{O}\right), 4.41\left(\mathrm{~s}, 1 \mathrm{H}, \mathrm{C}_{4}-\mathrm{H}\right), 4.85(\mathrm{~s}, 1 \mathrm{H}, \mathrm{NH})$, $7.02\left(\mathrm{t}, J=8.5 \mathrm{~Hz}, 1 \mathrm{H}\right.$, aromatic $\left.\mathrm{C}\left(5^{\prime}\right) \mathrm{H}\right), 7.11(\mathrm{t}, J=7.5 \mathrm{~Hz}, 1 \mathrm{H}$, aromatic $\left.\mathrm{C}\left(4^{\prime}\right) \mathrm{H}\right), 7.21\left(\mathrm{~d}, J=8.5 \mathrm{~Hz}, 1 \mathrm{H}\right.$, aromatic $\left.\mathrm{C}\left(6^{\prime}\right) \mathrm{H}\right)$, $7.37\left(\mathrm{~d}, J=7.5 \mathrm{~Hz}, 1 \mathrm{H}\right.$, aromatic $\left.\mathrm{C}\left(3^{\prime}\right) \mathrm{H}\right)$.

5-Benzyl 3-Methyl 4-(2-Chlorophenyl)-2,6-dimethyl-1,4dihydropyridine-3,5-dicarboxylate $(\mathbf{6 h})$

TLC purification afforded $0.601 \mathrm{~g}$ (81\% yield), pure oil, Anal. (\%): $\left(\mathrm{C}_{23} \mathrm{H}_{22} \mathrm{ClNO}_{4}\right)$, Calcd (Found), C: 67.07 (66.89), $\mathrm{H}: 5.38$ (5.47), N: 3.40 (3.36). ${ }^{1} \mathrm{H}-\mathrm{NMR}\left(500 \mathrm{MHz}, \mathrm{CDCl}_{3}\right) \delta$ : $2.27\left(\mathrm{~s}, 3 \mathrm{H}, \mathrm{COOCH}_{3}\right), 3.47\left(\mathrm{~s}, 6 \mathrm{H}, \mathrm{C}_{2}-\mathrm{CH}_{3}\right.$ and $\left.\mathrm{C}_{6}-\mathrm{CH}_{3}\right), 3.60$ $\left(\mathrm{s}, 2 \mathrm{H}, \mathrm{COOCH}_{2}\right), 4.98\left(\mathrm{~s}, 1 \mathrm{H}, \mathrm{C}_{4}-\mathrm{H}\right), 5.44(\mathrm{~s}, 1 \mathrm{H}, \overline{\mathrm{NH}}), 7.01$ $\left(\mathrm{t}, J=8.5 \mathrm{~Hz}, 1 \mathrm{H}, \mathrm{C}\left(5^{\prime}\right) \mathrm{H}\right), 7.08-7.15\left(\mathrm{~m}, 8 \mathrm{H}\right.$, aromatic $\mathrm{C}\left(4^{\prime}\right) \mathrm{H}$, $\mathrm{C}\left(6^{\prime}\right) \mathrm{H}, \mathrm{C}\left(3^{\prime}\right) \mathrm{H}$ and $\left.\mathrm{OCH}_{2} \underline{\mathrm{C}}_{6} \underline{\mathrm{H}}_{5}\right)$. 
5-Ethyl 3-Methyl 4-(3-Chlorophenyl)-2,6-dimethyl-1,4dihydropyridine-3,5-dicarboxylate (6i)

Recrystallization from methanol afforded $0.302 \mathrm{~g}$ (48\%), mp: 118-120 ${ }^{\circ} \mathrm{C}$, Anal. (\%): $\left(\mathrm{C}_{18} \mathrm{H}_{20} \mathrm{ClNO}_{4}\right)$, Calcd (Found), C: 61.80 (61.78), H: 5.76 (5.85), N: 4.00 (3.69). ${ }^{1} \mathrm{H}-\mathrm{NMR}$ (500 MHz, $\left.\mathrm{CDCl}_{3}\right) \delta: 1.09\left(\mathrm{t}, J=6.1 \mathrm{~Hz}, 3 \mathrm{H}, \mathrm{CH}_{2} \mathrm{CH}_{3}\right), 2.25\left(\mathrm{~s}, 6 \mathrm{H}, \mathrm{C}_{2}-\right.$ $\mathrm{CH}_{3}$ and $\left.\mathrm{C}_{6}-\mathrm{CH}_{3}\right), 3.61\left(\mathrm{~s}, 3 \mathrm{H}, \mathrm{COOCH}_{3}\right), 3.90-4.30(\mathrm{~m}, 2 \mathrm{H}$, $\left.\mathrm{COOCH}_{2}\right), 4.89\left(\mathrm{~s}, 1 \mathrm{H}, \mathrm{C}_{4}-\mathrm{H}\right), 5.66(\mathrm{~s}, 1 \mathrm{H}, \mathrm{NH}), 6.85-7.15(\mathrm{~m}$, $1 \mathrm{H}$, aromatic $\mathrm{C}\left(6^{\prime}\right) \mathrm{H}$ and $\left.\mathrm{C}\left(5^{\prime}\right) \mathrm{H}\right), 7.19(\mathrm{~d}, J=8.8 \mathrm{~Hz}, 1 \mathrm{H}$, aromatic $\left.\mathrm{C}\left(4^{\prime}\right) \mathrm{H}\right), 7.31$ (s, $\left.1 \mathrm{H}, \mathrm{C}\left(2^{\prime}\right) \mathrm{H}\right)$.

5-Isopropyl 3-Methyl 4-(3-Chlorophenyl)-2,6-dimethyl-1,4dihydropyridine-3,5-dicarboxylate $(\mathbf{6 j})$

Recrystallization from methanol afforded $0.257 \mathrm{~g}$ (39\%), mp: $133-135^{\circ} \mathrm{C}$, Anal. (\%): $\left(\mathrm{C}_{19} \mathrm{H}_{22} \mathrm{ClNO}_{4}\right)$, Calcd (Found), $\mathrm{C}: 62.72$ (62.63), H: 6.09 (5.83), N: 3.85 (4.16). ${ }^{1} \mathrm{H}-\mathrm{NMR}$ $\left(500 \mathrm{MHz}, \mathrm{CDCl}_{3}\right) \delta: 1.07\left(\mathrm{~d}, J=5.9 \mathrm{~Hz}, 3 \mathrm{H}, \mathrm{CH}\left(\mathrm{CH}_{3}\right)_{2}\right), 1.23$ $\left(\mathrm{d}, J=5.9 \mathrm{~Hz}, 3 \mathrm{H}, \mathrm{CH}\left(\underline{\mathrm{CH}}_{3}\right)_{2}\right), 2.24\left(\mathrm{~s}, 6 \mathrm{H}, \mathrm{C}_{2}-\underline{\mathrm{CH}}_{3}\right.$ and $\mathrm{C}_{6}-$ $\left.\mathrm{CH}_{3}\right), 3.62\left(\mathrm{~s}, 3 \mathrm{H}, \mathrm{COOCH}{ }_{3}\right), 4.78-5.13\left(\mathrm{~m}, 2 \mathrm{H}, \underline{\mathrm{CH}}\left(\mathrm{CH}_{3}\right)_{2}\right.$, $\left.\mathrm{C}_{4}-\mathrm{H}\right), 5.69(\mathrm{~s}, 1 \mathrm{H}, \mathrm{NH}), 7.04-7.22\left(\mathrm{~m}, 3 \mathrm{H}\right.$, aromatic $\mathrm{C}\left(6^{\prime}\right) \mathrm{H}$, $\mathrm{C}\left(5^{\prime}\right) \mathrm{H}$ and $\left.\mathrm{C}\left(4^{\prime}\right) \mathrm{H}\right), 7.28$ (s, $1 \mathrm{H}$, aromatic $\left.\mathrm{C}\left(2^{\prime}\right) \mathrm{H}\right)$.

3-Ethyl 5-Isopropyl 4-(3-Chlorophenyl)-2,6-dimethyl-1,4dihydropyridine-3,5-dicarboxylate (6k)

Recrystallization from methanol afforded $0.252 \mathrm{~g}$ (37\%), mp: $117-119^{\circ} \mathrm{C}$, Anal. (\%): $\left(\mathrm{C}_{20} \mathrm{H}_{24} \mathrm{ClNO}_{4}\right)$, Calcd (Found), $\mathrm{C}: 63.57$ (63.49), H: 6.40 (6.13), N: 3.71 (3.80). ${ }^{1} \mathrm{H}-\mathrm{NMR}$ $\left(500 \mathrm{MHz}, \mathrm{CDCl}_{3}\right) \delta: 0.87\left(\mathrm{~d}, J=5.9 \mathrm{~Hz}, 3 \mathrm{H}, \mathrm{CH}\left(\mathrm{CH}_{3}\right)_{2}\right), 0.90$ (d, $\left.J=5.9 \mathrm{~Hz}, 3 \mathrm{H}, \mathrm{CH}\left(\mathrm{CH}_{3}\right)_{2}\right), 1.88\left(\mathrm{t}, J=6.1 \mathrm{~Hz}, 3 \mathrm{H}, \mathrm{CH}_{2} \mathrm{CH}_{3}\right)$, $2.34\left(\mathrm{~s}, 6 \mathrm{H}, \mathrm{C}_{2}-\underline{\mathrm{CH}}_{3}\right.$ and $\left.\mathrm{C}_{6}-\mathrm{CH}_{3}\right), 3.72-3.88\left(\mathrm{~m}, 3 \mathrm{H}, \mathrm{COOCH}_{2}\right.$, $\mathrm{COOCH}), 5.01\left(\mathrm{~s}, 1 \mathrm{H}, \mathrm{C}_{4}-\mathrm{H}\right), 5.71(\mathrm{~s}, 1 \mathrm{H}, \mathrm{NH}), 6.92-7.16(\mathrm{~m}$, $2 \mathrm{H}$, aromatic $\mathrm{C}\left(6^{\prime}\right) \mathrm{H}$ and $\left.\mathrm{C}\left(5^{\prime}\right) \mathrm{H}\right), 7.19(\mathrm{~d}, J=8.8 \mathrm{~Hz}, 1 \mathrm{H}$, aromatic $\left.\mathrm{C}\left(4^{\prime}\right) \mathrm{H}\right), 7.28\left(\mathrm{~s}, 1 \mathrm{H}\right.$, aromatic $\left.\mathrm{C}\left(2^{\prime}\right) \mathrm{H}\right)$.

5-Isobutyl 3-Methyl 4-(3-Chlorophenyl)-2,6-dimethyl-1,4dihydropyridine-3,5-dicarboxylate (61)

Recrystallization from methanol afforded $0.166 \mathrm{~g}$ (24\%), mp: 96-98 ${ }^{\circ} \mathrm{C}$, Anal. (\%): $\left(\mathrm{C}_{20} \mathrm{H}_{24} \mathrm{ClNO}_{4}\right)$, Calcd (Found), C: 63.57 (63.75), H: 6.40 (6.23), N: 3.71 (3.91). ${ }^{1} \mathrm{H}-\mathrm{NMR}(500 \mathrm{MHz}$, $\left.\mathrm{CDCl}_{3}\right) \delta: 0.80\left(\mathrm{~d}, J=6.4 \mathrm{~Hz}, 6 \mathrm{H}, \mathrm{CH}\left(\mathrm{CH}_{3}\right)_{2}\right), 1.83-1.93(\mathrm{~m}$, $\left.1 \mathrm{H}, \underline{\mathrm{CH}}\left(\mathrm{CH}_{3}\right)_{2}\right), 2.28\left(\mathrm{~s}, 6 \mathrm{H}, \mathrm{C}_{2}-\mathrm{CH}_{3}\right.$ and $\left.\mathrm{C}_{6}-\mathrm{CH}_{3}\right), 3.61$ (s, $\left.3 \mathrm{H}, \overline{\mathrm{COOCH}}_{3}\right), 3.79$ (d, J=6.5 Hz, $\left.1 \mathrm{H}, \mathrm{COOCH}_{2}\right), 3.85$ (d, $\left.J=6.5 \mathrm{~Hz}, 1 \mathrm{H}, \mathrm{COOCH}_{2}\right) 4.91\left(\mathrm{~s}, 1 \mathrm{H}, \mathrm{C}_{4}-\mathrm{H}\right), 5.74(\mathrm{~s}, 1 \mathrm{H}, \mathrm{NH})$, 7.01-7.22 (m, 3H, aromatic $\mathrm{C}\left(6^{\prime}\right) \mathrm{H}, \mathrm{C}\left(5^{\prime}\right) \mathrm{H}$ and $\left.\mathrm{C}\left(4^{\prime}\right) \mathrm{H}\right), 7.28$ (s, $1 \mathrm{H}$, aromatic $\left.\mathrm{C}\left(2^{\prime}\right) \mathrm{H}\right)$.

3-Methyl 5-(2-Methoxyethyl) 4-(3-Chlorophenyl)-2,6-dimethyl-1,4-dihydropyridine-3,5-dicarboxylate (6m)

Recrystallization from methanol afforded $0.198 \mathrm{~g}$ (29\%), mp: $106-108^{\circ} \mathrm{C}$, Anal. (\%): $\left(\mathrm{C}_{19} \mathrm{H}_{22} \mathrm{ClNO}_{5}\right)$, Calcd (Found), $\mathrm{C}: \quad 60.08$ (59.98), H: 5.84 (5.55), N: 3.69 (3.39). ${ }^{1} \mathrm{H}-\mathrm{NMR}$ $\left(500 \mathrm{MHz}, \mathrm{CDCl}_{3}\right) \delta: 2.28\left(\mathrm{~s}, 6 \mathrm{H}, \mathrm{C}_{2}-\mathrm{CH}_{3}\right.$ and $\left.\mathrm{C}_{6}-\mathrm{CH}_{3}\right), 3.33$ (s, $\left.3 \mathrm{H}, \mathrm{OCH}_{3}\right), 3.45-3.55\left(\mathrm{~m}, 2 \mathrm{H}, \mathrm{COOCH}_{2} \mathrm{CH}_{2} \mathrm{O}\right), 3.63(\mathrm{~s}, 3 \mathrm{H}$, $\left.\mathrm{COOCH}_{3}\right), 4.03-4.35\left(\mathrm{~m}, 2 \mathrm{H}, \mathrm{COOCH}_{2} \mathrm{CH}_{2} \mathrm{O}\right), 5.01(\mathrm{~s}, 1 \mathrm{H}$, $\left.\mathrm{C}_{4}-\mathrm{H}\right), 5.79(\mathrm{~s}, 1 \mathrm{H}, \mathrm{NH}), 7.01-7.22\left(\mathrm{~m}, 3 \mathrm{H}\right.$, aromatic $\mathrm{C}\left(6^{\prime}\right) \mathrm{H}$, $\mathrm{C}\left(5^{\prime}\right) \mathrm{H}$ and $\left.\mathrm{C}\left(4^{\prime}\right) \mathrm{H}\right), 7.26\left(\mathrm{~s}, 1 \mathrm{H}\right.$, aromatic $\left.\mathrm{C}\left(2^{\prime}\right) \mathrm{H}\right)$.

3-Ethyl 5-(2-Methoxyethyl) 4-(3-Chlorophenyl)-2,6-dimethyl-1,4-dihydropyridine-3,5-dicarboxylate (6n)

Recrystallization from methanol afforded $0.203 \mathrm{~g}$ (29\%), mp: $116-118^{\circ} \mathrm{C}$, Anal. (\%): $\left(\mathrm{C}_{20} \mathrm{H}_{24} \mathrm{ClNO}_{5}\right)$, Calcd (Found), C: 60.99 (61.00), H: 6.14 (6.36), N: 3.56 (3.88). ${ }^{1} \mathrm{H}-\mathrm{NMR}$ $\left(500 \mathrm{MHz}, \mathrm{CDCl}_{3}\right) \delta: 1.16\left(\mathrm{t}, J=6.1 \mathrm{~Hz}, 3 \mathrm{H}, \mathrm{CH}_{2} \mathrm{CH}_{3}\right), 2.29$ (s, 6H, $\mathrm{C}_{2}-\mathrm{CH}_{3}$ and $\left.\mathrm{C}_{6}-\underline{\mathrm{CH}}_{3}\right), 3.34\left(\mathrm{~s}, 3 \mathrm{H}, \mathrm{OCH}_{3}\right), 3.52(\mathrm{t}$, $\left.J=4.5 \mathrm{~Hz}, 2 \mathrm{H}, \mathrm{COOCH}_{2} \mathrm{CH}_{2} \mathrm{O}\right), 4.07-4.15\left(\mathrm{~m}, 2 \mathrm{H}, \mathrm{CH}_{2} \mathrm{CH}_{3}\right)$, $4.20\left(\mathrm{t}, J=4.5 \mathrm{~Hz}, 2 \mathrm{H}, \mathrm{COOCH}_{2} \mathrm{CH}_{2} \mathrm{O}\right), 4.98\left(\mathrm{~s}, 1 \overline{\mathrm{H}}, \mathrm{C}_{4}-\mathrm{H}\right)$, $5.71(\mathrm{~s}, 1 \mathrm{H}, \mathrm{NH}), 7.04-7.15\left(\mathrm{~m}, 2 \mathrm{H}\right.$, aromatic $\mathrm{C}\left(6^{\prime}\right) \mathrm{H}$ and $\left.\mathrm{C}\left(5^{\prime}\right) \mathrm{H}\right), 7.19\left(\mathrm{~d}, J=8.8 \mathrm{~Hz}, 1 \mathrm{H}\right.$, aromatic $\left.\mathrm{C}\left(4^{\prime}\right) \mathrm{H}\right), 7.27(\mathrm{~s}, 1 \mathrm{H}$, aromatic $\left.\mathrm{C}\left(2^{\prime}\right) \mathrm{H}\right)$

Pharmacology Male guinea-pigs weighing 300-450g were sacrificed and the intestine was removed above the ileocecal junction and longitudinal smooth muscle segments of $2 \mathrm{~cm}$ length were mounted under a resting tension of $0.5 \mathrm{~g}$. The segments were maintained at $37^{\circ} \mathrm{C}$ in a $10 \mathrm{~mL}$ jacketed organ bath (ML870B6/C, PanLab). The organ bath was filled with a physiological saline solution of the following composition (mmol): $\mathrm{NaCl}$ (137), $\mathrm{CaCl}_{2}$ (1.8), $\mathrm{KCl}$ (2.7), $\mathrm{MgSO}_{4}$ (1.1), $\mathrm{NaH}_{2} \mathrm{PO}_{4}(0.4), \mathrm{NaHCO}_{3}(12)$ and glucose (5), and was continuously aerated with carbogen (oxygen-carbon dioxide; 95:5) at $37^{\circ} \mathrm{C}$. The muscles were equilibrated for $1 \mathrm{~h}$ with a solution changed every $15 \mathrm{~min}$. The contractions were recorded with a force displacement transducer (MLT0201, PanLab) connected to amplifier. Stocks of the tested compounds $\left(10^{-6} \mathrm{M}\right.$ in ethanol) were stored protected from light. Dilutions were made with double distilled water.

In order to study the effects of synthesized DHP on KClinduced contraction of ileum, at the first step, several contractions with $\mathrm{KCl}(40 \mathrm{~mm})$ were made. No significant differences between $\mathrm{KCl}$-induced contractions were considered as stability of tissue and thereafter the main experiments were started. At this step, $\mathrm{KCl}(40 \mathrm{~mm})$-elicited contraction was recorded again and the peak of the first phase (phasic contraction) was considered as a control. The contractile response was taken as the $100 \%$ value for the tonic (slow) component of the response. Then, tested compounds were cumulatively added and compound induced relaxation of contracted muscle was expressed as percent of control.

The $\mathrm{IC}_{50}$ of each compound (molar concentration needed to produce $50 \%$ relaxation on contracted ileal smooth muscle) were graphically determined from the concentration-response curves. Each segment was treated with only one compound. Nifedipine was used as reference compound. A triplicate experiment was performed for each compound. This protocol was approved by Tanta University ethics committee.

Statistic Data Analysis Data were statistically analyzed using SPSS 21.0 program (SPSS, Chicago, IL, U.S.A.). The results are recorded as the mean \pm S.E.M. and they were evaluated statistically using one-way ANOVA following post hoc test.

Molecular Docking Docking process requires the $3 \mathrm{D}$ structure of both protein and ligands. Coordinates of the DHP receptor model developed by Shaldam et al. ${ }^{33)}$ were used. All ligands were drawn into Marvin Sketch V5.1.3. ${ }^{35)}$ The most energetically favored conformer was saved as (*.mol2) file format for docking. The optimal geometry of the ligands was determined during the docking process. Docking was performed in a multistep procedure using Molegro Virtual Docker V6.0 program. Firstly, nifedipine was placed in the proposed active site defined by SAR. ${ }^{33)}$ The NH-group of DHP is considered as the most crucial part of the ligand that forms $\mathrm{H}$-bond with the receptor. Consequently, docking template was generated in attempts to align all ligands to the orientation of nifedipine. This is a demanding to bias the formation of H-bond with Tyr $^{1149}$ — an important DHP sensing residue-while takes the orientation described by SAR. ${ }^{33)}$ Docking template consisted of one hydrogen bond donor, two rings, three hydrogen bond acceptors and five steric moieties. Iterative simplex algorithm was chosen to perform docking process with 15 runs per 
ligand, 50 population size, $100 \mathrm{max}$ iteration and 8 poses for each ligand. Secondly, MolDock docking engine ${ }^{36)}$ using docking template and the optimized ligands was executed. Finally, the top returned poses were manually modified to maximize binding to DHP sensing residues.

\section{Conclusion}

The lipophilicity of the substituent at 4-position of DHP ring is an important factor that may increase the activity of the calcium antagonist. On the other hand, this substituent should not be too bulky to interfere with ring to ring hydrophobic interaction with $\mathrm{Tyr}^{1460}$. Consequently, this interference makes increasing the length of ester side chain is not an effective approach to increase the calcium antagonist activity. The presence of chelating group substituent at 4-position of DHP ring may guarantee the ring to ring hydrophobic interaction and chelates the $\mathrm{Ca}^{2+}$ cofactor making more binding and blocking effect to the receptor. The presence of substituent at ortho- or meta-position of 4-phenyl ring is essential to accommodate a non-coplanar relationship between phenyl and DHP rings which is an essential requirement for binding to the active site of the receptor.

Acknowledgment All authors thank the faculty of pharmacy, Tanta University for financial support.

Conflict of Interest The authors declare no conflict of interest.

\section{References}

1) Tang L., Gamal El-Din T. M., Payandeh J., Martinez G. Q., Heard T. M., Scheuer T., Zheng N., Catterall W. A., Nature (London), 505, 56-61 (2014)

2) Zamponi G. W., Drug Dev. Res., 42, 131-143 (1997).

3) Dolphin A. C., Br. J. Pharmacol., 147 (Suppl. 1), S56-S62 (2006).

4) Gullapalli S., Ramarao P., Neuropharmacology, 42, 467-475 (2002).

5) Zhorov B. S., Folkman E. V., Ananthanarayanan V. S., Arch. Biochem. Biophys., 393, 22-41 (2001).

6) Peterson B. Z., Catterall W. A., J. Biol. Chem., 270, 18201-18204 (1995).

7) Kappe C., Molecules, 3, 1-9 (1998).

8) Swarnalatha G., Prasanthi G., Sirisha N., Madhusudhana Chetty C., International Journal of ChemTech Research, 3, 75-89 (2011).

9) Breitenbucher J. G., Figliozzi G., Tetrahedron Lett., 41, 4311-4315 (2000).
10) Boer R., Gekeler V., Drugs Future, 20, 499-509 (1995).

11) Briukhanov V. M., Zverev IaF., Elkin V. I., Eksp. Klin. Farmakol., 57, 47-49 (1994).

12) Bahekar S. and Shinde D., Anglais, 52, 281-287 (2002).

13) Wächter G. A., Davis M. C., Martin A. R., Franzblau S. G., J. Med. Chem., 41, 2436-2438 (1998).

14) Sunkel C. E., de Casa-Juana M. F., Santos L., Gomez M. M., Villarroya M., Gonzalez-Morales M. A., Priego J. G., Ortega M. P., J. Med. Chem., 33, 3205-3210 (1990).

15) Cooper K., Fray M. J., Parry M. J., Richardson K., Steele J., J. Med. Chem., 35, 3115-3129 (1992).

16) Triggle D. J., Eur. J. Pharmacol., 375, 311-325 (1999).

17) Triggle D. J., Cell. Mol. Neurobiol., 23, 293-303 (2003).

18) Hatano N., Ohya S., Muraki K., Giles W., Imaizumi Y., Br. J. Pharmacol., 139, 533-544 (2003).

19) Dhein S., Salameh A., Berkels R., Klaus W., Drugs, 58, 397-404 (1999).

20) Katoh H., Schlotthauer K., Bers D. M., Circ. Res., 87, 106-111 (2000).

21) Hemmateenejad B., Miri R., Akhond M., Shamsipur M., Arch. Pharm., 335, 472-480 (2002).

22) Navidpour L., Shafaroodi H., Miri R., Dehpour A. R., Shafiee A., Il Farmaco., 59, 261-269 (2004).

23) Miri R., Javidnia K., Sarkarzadeh H., Hemmateenejad B., Bioorg. Med. Chem., 14, 4842-4849 (2006).

24) El-Moselhy T. F., Chem. Biol. Interact., 3, 123-136 (2013).

25) Hantzsch A., Justus Liebigs Ann. Chem., 215, 1-82 (1882).

26) Meyer H., Bossert F., Wehinger E., Stoepel K., Vater W., Arzneimittelforschung, 31, 407-409 (1981).

27) Triggle C. R., Swamy V. C., Triggle D. J., Can. J. Physiol. Pharmacol., 57, 804-818 (1979).

28) Shafiee A., Pirouzzadeh B., Ghasemian F., Parang K., J. Heterocycl. Chem., 29, 1021-1023 (1992).

29) Miri R., Howlett S. E., Knaus E. E., Arch. Pharm., 330, 290-294 (1997).

30) Daryabari N., Akbarzadeh T., Amini M., Miri R., Mirkhani H., Shafiee A., JICS, 4, 30-36 (2007).

31) Meier K., Knepel W., Schofl C., Endocrinology, 122, 2764-2770 (1988).

32) Brooks D. D., Huddart H., Lennard R and Hill R. B., J. Exp. Biol., 149, 379-394 (1990)

33) Shaldam M. A., Elhamamsy M. H., Esmat E. A., El-Moselhy T. F., ISRN Med. Chem., 2014, 14 (2014).

34) El-Moselhy T. F., Alexandria J. Pharm. Sci., 16, 35-39 (2002).

35) "ChemAxon." Marvin 5.11.5, 2013, ChemAxon: http://www.chemaxon. com

36) Thomsen R., Christensen M. H., J. Med. Chem., 49, 3315-3321 (2006). 